\section{FAMILY CARERS' EXPERIENCES DURING THE END OF LIFE CARE IN A RESOURCE POOR SETTING}

${ }^{1}$ Dalhat Sani Khalid, ${ }^{2}$ Gina Higginbottom, ${ }^{2}$ Christopher Bailey. ${ }^{1}$ Department of Nursing Sciences, Ahmadu Bello University, Zaria-Nigeria; ${ }^{2}$ School of Health Sciences, University of Nottingham

\subsection{6/bmjspcare-2018-ASPabstracts.63}

Background Palliative and end of life care is an international issue but is receiving very little formal attention in SubSaharan Africa in general and Nigeria in particular, there is inadequate information and lack of accessibility to care. Research to inform adequate end of life care in Africa is at an early stage and many research areas need to be explored. Previous studies were carried out in western countries, but the information is culturally in-congruent and not transferable to poor resource context like Nigeria. This means that the information exists will not necessarily be helpful because of the cultural sensitivity, values, and beliefs attached to end of life care in Nigeria and other Sub-Saharan African countries. The study explored the experiences of family carers during the end of life care.

Method An ethnographic case study approach was used to explore family carers' experiences during the end of life care in the medical unit of a Nigerian University Teaching hospital. Interviews and participant observations were used as the tools for data collection.

Results Fourteen family carers were interviewed separately, and each interview was preceded by a week of observations. Preliminary analysis identified five overarching themes: Challenges/barriers to end of life care, Family carers experiences, Educational needs and support, Family carers roles and Family carers' perception about end of life care.

Conclusion Majority of the family carers described their end of life care experiences as unpleasant, there was lack of support generally and a wide communication gap between carers and healthcare professionals. The study generates evidencebased clinical and health services data to inform policy, practice and future research. At the same time to ensure that end of life care service provision in Nigeria is effective, appropriate, acceptable, ethical and culturally competent.

\section{A PILOT AUDIT TO ASSESS ACHIEVEMENT OF 'PREFERRED PLACE OF DEATH' (PPD) FOR CANCER AND NON-CANCER PATIENTS}

Anitia Brigham, Janet Gillett, Catherine Leask. St. Margaret's Hospice, Somerset

\subsection{6/bmjspcare-2018-ASPabstracts.64}

Background Achievement of 'preferred place of death' (PPD) has been adopted as a key performance indicator for quality in end of life care. Approximately $60 \%-70 \%$ of people report they wish to die at home, however, evidence shows that place of death is actually only 7 th on the list of priorities for ' $a$ good death'. The aim of this audit was to evaluate the proportion of patients who achieved their PPD; and if not, why this was not attainable. It also assessed if diagnosis made an impact on the achievement of PPD.

Methods Retrospective case note review of the last 50 cancer and 50 non-cancer deaths prior to 1 st October 2015 where a PPD was documented in the hospice electronic record. Information regarding the events and care prior to death was sourced from hospice, GP, hospital and district nursing records.

Results 67/100 (67\%) of patients stated home as their PPD. $35 / 50(70 \%)$ cancer and 40/50 (80\%) non-cancer patients achieved their PPD. Of the 25 patients who did not achieve their PPD, there were 3 categories of reasons for this:

- Unforeseen circumstances (68\%) e.g. uncontrolled care or symptom needs, rapid deterioration preventing transfer, ongoing active treatment;

- System limitations (24\%) e.g. bed or care package unavailability;

- Missed opportunities for implementation of anticipatory measures (8\%) e.g. no 'just in case' box, poor communication between care settings.

Conclusions 3 out of 4 patients achieved their PPD. Diagnosis did not appear to significantly impact whether PPD was achieved. Where PPD was not achieved, unforeseen circumstances was the most common reason.

Recommendations

- Discussions about PPD should include exploration of unforeseen circumstances.

- Patients' overall priorities for care should be identified in conjunction with PPD discussions.

- PPD achievement should be avoided as a marker of quality care.

\section{COMMUNICATION AND ADVANCED CARE PLANNING IN} A GENERAL PRACTICE SETTING

\section{A Mondon-Ballantyne. East Kent NHS Foundation Trust}

\subsection{6/bmjspcare-2018-ASPabstracts.65}

Aims To investigate the effective undertaking and recording of end of life conversations within a busy urban general practice. These included important areas such as physical, psychological, social, spiritual and financial concerns as well as DNAR decisions and preferred place of care.

Methods Utilising EMIS database, all deaths between the 1 st January 2016 and 31 st December 2016 of patients registered at Newington Road Surgery, Ramsgate were identified. The electronic notes and records were accessed and reviewed. A total of 63 deaths were recorded.

Results In 54\% of deaths in the practice, end of life discussions would have been appropriate because of evidence of general decline, end stage cancer or frailty. Despite this only $38 \%$ of these patients had end of life discussions.

Out of these discussions $62 \%$ focused on physical symptoms with only $38 \%$ recording psychological, social, financial or spiritual discussions.

Most surprisingly only 9\% of patients had a saved and accessible care plan and none of those plans recorded had any information regarding patient's preferred place of care, preferred place of death or an escalation plan regarding hospital admissions.

Conclusions This project concluded that many factors are involved when contributing to discussions had by GPs towards the end of patient's lives. These included time pressures and the need for additional input from hospital, oncology and palliative care teams.

However, this project highlighted the importance in identifying patients towards the end of their life in the community 
(and the use of approved tools such as SPICT) and the need for increased education for GPs about all aspects of supportive end of life care and advanced care planning.

\section{THE COST OF A NIGHT NURSING SERVICE AT RENNIE GROVE HOSPICE CARE, AND THE TOTAL COMMUNITY COST OF CARE AT HOME COMPARED TO AN ADMISSION TO HOSPITAL}

S Varvel, SG Spiro, A Graham, J Sixsmith, A Ward. Rennie Grove Hospice Care, The University of Northampton, The University of Dundee

\subsection{6/bmjspcare-2018-ASPabstracts.66}

Background Rennie Grove (RG) runs a 24/7 Hospice at Home service. An independent study calculated the cost of a visit and the total community cost of home care, including all health care professional (HCP), carer, and family member visits.

Methods Over a period of 145 days, 550 calls and 335 visits made to/by the night team were recorded, averaging 3.79 per night. The salary cost per hour for each nurse, plus organisational add on costs, were calculated. To derive a total community cost, 35 families, able to consent, kept a diary for up to two-weeks, recording all HCP, carer and family support visits and duration of each visit. 17 diaries were returned. Descriptive analysis was used with the Statistical Package for the Social Sciences (SPSS v22). Costs were taken for HCPs and social care services from the PSSRU data 2015/2016.

Results Average length of the diaries was 10.4 days. For 3.79 visits per night the cost per visit was $£ 195$ (RG nurses travel in pairs). 17 patient diaries covered 177 days and showed a range of visits and complexity of care. RG staff provided $19 \%$ of the care at a cost of $£ 3,295$; district nurses $13 \%$, cost $£ 2,005$; formal carers $55 \%$ at a cost of $£ 1344$. 23 GPs visits comprised $4 \%$ of visits but $15 \%$ of total costs. MacMillan/Marie Curie nurses accounted for just 2\% of visits but $19 \%$ of cost as they stayed overnight. The cost of 177 days of care for 17 patients was $£ 11,814$; i.e. $£ 66.7$ per day as care was not needed every day of each diary period.

Conclusions The cost of home care seems acceptable, compared to the national average cost of a day in an inpatient specialist palliative care bed at $£ 397-£ 400$ (Data.Gov.UK, 2015).

\section{HOW SATISFIED ARE CARERS/FAMILIES WITH A NIGHT TEAM SERVICE AS PART OF 24/7 HOSPICE AT HOME CARE?}

S Varvel, A Ward, J Sixsmith, A Graham, SG Spiro. Rennie Grove Hospice Care, The University of Northampton, The University of Dundee

\subsection{6/bmjspcare-2018-ASPabstracts.67}

Background Rennie Grove (RG) runs a 24/7 service with a dedicated responsive night team. Through an independent study, carers' levels of service satisfaction with the night team were investigated.

Methods A questionnaire was sent to carers $(n=268)$ who had a relative die under the care of RG within the last 6 weeks to one year, 84 completed questionnaires were returned. Follow-up face-to-face semi-structured interviews were carried out with carers $(n=18)$. Quantitative data was analysed using SPSS v 22, while qualitative data was thematically analysed.

Results The carer questionnaire reported that 71\% thought the speed of the response by the night team was fast. Overall satisfaction with telephone call response was high with $82 \%$ very satisfied. Carers also agreed strongly that the telephone calls provided good quality of care (90\%), good follow-up care $(88 \%)$, that they were given sufficient time $(88 \%)$, and received useful advice (83\%). For a visit, 93\% of carers were highly satisfied and $7 \%$ satisfied. Carers strongly agreed that they were given sufficient time (90\%), and were confident in the care provided (90\%), care was useful (87\%) and followup was good (84\%), worries were reduced (81\%) and they had a say in decisions made (81\%). During the interviews, carers described the night service as: supportive, amazing, caring, reassuring, professional, kind and compassionate, excellent, loving, calm. Carers perceived the service to be holistic and rated the service highly for supporting them to ensure family members could be cared for at home, as preferred. For comparison, of those dying in hospital, only $46 \%$ were highly satisfied. Carer wellbeing scores were lower than the national average, but reported similar levels of anxiety.

Conclusions A night team service can provide excellent, reassuring and holistic care that meets the care needs and wishes of the patient and family.

\section{DOES A 24/7 HOSPICE AT HOME SERVICE PREVENT OR POSTPONE ACUTE HOSPITAL ADMISSIONS?}

SG Spiro, A Ward, A Graham, J Sixsmith. Rennie Grove Hospice Care, The University of Northampton, The University of Dundee

\subsection{6/bmjspcare-2018-ASPabstracts.68}

Background Most people wish to die at home but in England almost 50\% die in hospital, suggesting that more can be done to keep people at home. Some studies have shown this may be possible, especially with adequate support and good pain control.

Methods Rennie Grove Hospice Care carried out an independent study to identify the value of their night team in providing 24/7 care over a period of 145 nights. The study considered whether the provision of overnight care affected decisions to make hospital admissions. Data from a night nurse template recording all overnight visits, a review of 42 patient/carer records of those who had called the night team, a carer questionnaire $(n=87)$, carer interviews $(n=18)$ and staff interviews $(n=9)$, were analysed.

Results The night nurse template recorded 5 overnight admissions made during the study period, all for acute reasons and considered unavoidable. Review of 42 patient records showed 23 hospital/hospice admissions occurred, most during the daytime (18 to hospital, 5 to a hospice) of which only 3 were considered avoidable. The carer questionnaires showed 13 (16\%) of their cared for persons had been admitted to hospital in the last few weeks of life, with most reporting the admission was necessary. Staff interviews identified a pride in supporting patients to stay at home, while information from carers gave a clear indication that Rennie Grove nurses enabled their cared for individual to remain at home; that contact with the night team helped prevent or postpone an admission; and without Rennie Grove their alternative would have been 111/999, district nurse or out-of-hours GP. 